\title{
Testing the effectiveness and implementation of a brief version of the Common Elements Treatment Approach (CETA) in Ukraine: a study protocol for a randomized controlled trial
}

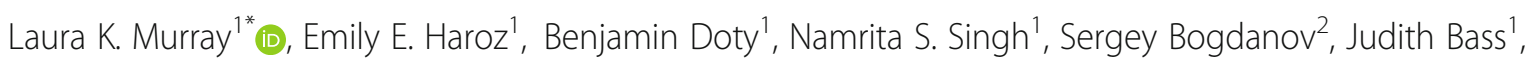
Shannon Dorsey ${ }^{3}$ and Paul Bolton ${ }^{4}$

\begin{abstract}
Background: Mental illness is a major public health concern. Despite progress understanding which treatments work, a significant treatment gap remains. An ongoing concern is treatment length. Modular, flexible, transdiagnostic approaches have been offered as one solution to scalability challenges. The Common Elements Treatment Approach (CETA) is one such approach and offers the ability to treat a wide range of common mental health problems. CETA is supported by two randomized trials from low- and middle-income countries showing strong effectiveness and implementation outcomes.
\end{abstract}

Methods/design: This trial evaluates the effectiveness and implementation of two versions of CETA using a noninferiority design to test two primary hypotheses: (1) a brief five-session version of CETA (Brief CETA) will provide similar effectiveness for reducing the severity of common mental health problems such as depression, post-traumatic stress, impaired functioning, anxiety, and substance use problems compared with the standard 8-12-session version of CETA (Standard CETA); and (2) both Brief and Standard CETA will have superior impact on the outcomes compared to a waitlist control condition. For both hypotheses, the main effect will be assessed using longitudinal data and mixed-effects regression models over a 6-month period post baseline. A secondary aim includes exploration of implementation factors. Additional planned analyses will include exploration of: moderators of treatment impact by disorder severity and comorbidity; the impact of individual therapeutic components; and trends in symptom change between end of treatment and 6-month assessment for all participants.

Discussion: This trial is the first rigorous study comparing a standard-length (8-12 sessions) modular, flexible, transdiagnostic, cognitive-behavioral approach to a shortened version of the approach (five sessions). Brief CETA entails "front-loading" with elements that research suggests are strong mechanisms of change. The study design will allow us to draw conclusions about the effects of both Brief and Standard CETA as well as which elements are integral to their mechanisms of action, informing future implementation and fidelity efforts. The results from this trial will inform future dissemination, implementation and scale-up of CETA in Ukraine and contribute to our understanding of the effects of modular, flexible, transdiagnostic approaches in similar contexts.

(Continued on next page)

\footnotetext{
* Correspondence: Imurra15@jhu.edu

${ }^{1}$ Department of Mental Health, Johns Hopkins Bloomberg School of Public

Health, 624 N. Broadway, Baltimore, MD 21205, USA

Full list of author information is available at the end of the article
}

(c) The Author(s). 2018 Open Access This article is distributed under the terms of the Creative Commons Attribution 4.0 International License (http://creativecommons.org/licenses/by/4.0/), which permits unrestricted use, distribution, and reproduction in any medium, provided you give appropriate credit to the original author(s) and the source, provide a link to the Creative Commons license, and indicate if changes were made. The Creative Commons Public Domain Dedication waiver (http://creativecommons.org/publicdomain/zero/1.0/) applies to the data made available in this article, unless otherwise stated. 
(Continued from previous page)

Trial registration: ClinicalTrials.gov, ID: NCT03058302 (U.S. National Library of Medicine). Registered on 20 February 2017.

Keywords: Global mental health, Transdiagnostic, Depression, Veterans, Internally displaced persons, Trauma, Cognitivebehavioral therapy, Implementation research

\section{Background}

Mental, neurological, and substance use disorders account for $10.4 \%$ of the global burden of disease [1]. The field of global mental health has made significant advances over the past decade in understanding the effectiveness of various evidence-based mental health treatments in low- and middle-income countries (LMIC) [2-10]. Despite this progress, the availability of such evidence-based treatments is limited by multiple challenges around buy-in, scale-up, and sustainability. Research shows that most individuals with mental health needs do not receive care - creating a substantial mental health "treatment gap" [11-13].

One possible strategy for addressing the challenge of scale-up is utilizing a transdiagnostic approach, in which providers receive training in one approach that can address multiple common mental health problems and comorbidity [14]. The use of transdiagnostic approaches may prevent the need to train providers in multiple focal treatments, which creates challenges for scale-up in terms of time and cost, and has limited ability to address comorbidities. Various transdiagnostic models, some modular, initially were developed and tested in high-income settings, and have demonstrated effectiveness and provider appeal [15-18]. The term transdiagnostic is widely used with varying underlying conceptualizations. In their conceptualization, Sauer-Zavala and colleagues [19] describe three approaches: (1) a universally applied principles approach, which is based on a school of thought (e.g., psychodynamic, cognitive-behavioral, humanistic) that is then applied to multiple disorders regardless of symptom presentation; (2) a modular or common-elements approach, which involves using components or elements of existing evidence-based treatments that can be delivered in different orders and combinations to address symptom presentation and wide-ranging comorbidities; and (3) a shared mechanism approach, which is informed by theory and targets underlying processes of certain disorders. Other potential differences across transdiagnostic treatments, regardless of how they are conceptualized, include more structural aspects such as dosage or intensity (e.g., number of sessions), decomposability or modularity (the degree to which elements are mostly independent of one another), flexibility, linearity, and whether or not they are multi-problem [20].

Building on growing empirical support for transdiagnostic treatments and their potential promise for efficiency, our research team developed The Common Elements Treatment Approach (CETA) [21], a modular, flexible, transdiagnostic approach specifically for LMIC, in which task-sharing is used, and providers often have minimal or no formal mental health training. CETA was modeled on a treatment developed by Drs. John Weisz and Bruce Chorpita [18, 22-25], specifically to be a modular, flexible, transdiagnostic approach as that was believed to have the most potential to address implementation and scale-up barriers in LMIC. Such transdiagnostic models could address a wide range of comorbid problems across the life span (reducing the number of treatments needed), with the ability to only deliver the dosage needed (potentially reducing cost and resources). The goal in adapting an existing intervention-versus just using the existing intervention-was to create a more simplified approach that had a limited number of elements, simple language, a short manual with practical step-sheets, and decision rules giving providers and their supervisors flexible choices in element selection, sequencing, and dose. The decision rules help the provider use the client's initial assessment (e.g., primary presenting problem; comorbidity) and ongoing presentation (e.g., symptom changes during treatment) to plan and adjust treatment as needed [21]. Our team has evaluated CETA effectiveness with adults in Iraq and Thailand, demonstrating that providers with minimal or no formal mental health training could provide CETA with fidelity and achieve positive outcomes with their clients (large effect sizes across outcomes) [2, 26].

Although the field of global mental health has a number of evidence-based mental health treatments with evidence of effectiveness, treatment duration is often cited as a significant barrier to scale-up and reduction of the treatment gap as the longer the treatment, the more staff and resources are needed, and the potential for dropout increases. Previous studies of cognitive-behavioral therapy (CBT)-based interventions, interpersonal psychotherapy, and CETA in LMIC have been tested with treatment durations of approximately 8-12 1 hour sessions [3, 7, 9, 26-28]. Scale-up challenges have led to a push to develop intervention models that are even briefer, particularly for highly mobile populations, such as trauma-affected groups or displaced persons [29]. A modular, flexible, transdiagnostic model provides an opportunity to decrease the length of treatment based on need (e.g., utilizing fewer elements), but still catering to 
the comorbid problems experienced by any population or individual.

There may be different methods to reducing the length of treatments. One approach to developing shorter interventions is to focus on elements or skills that may be easier to learn. Another approach is to identify treatment elements that seem to be essential, and, when treatments have been subjected to dismantling studiesaccount for greater variance in client outcomes. For example, although cognitive elements may be more challenging for lay providers and/or clients to understand and master, cognitive elements are also among those most frequently used in many evidence-based treatments for common mental health problems. Research suggests that cognitive interventions can be learned by lay counselors when provided with coaching and supervision $[3,9,26,28]$, and given their broad use in a number of evidence-based protocols may represent an important mechanism of action. Interventions using cognitive skills have been tested in LMIC and have shown evidence of effectiveness, often with large effect sizes. For example, in a study focused on survivors of sexual violence in Democratic Republic of Congo, Bass and colleagues [3] evaluated Cognitive Processing Therapy - Cognitive Therapy, which is an intervention that focuses primarily on cognitive elements. Effect sizes were greater than 1.0 for post-traumatic stress disorder (PTSD) and combined depression and anxiety. Trials in LMIC that used evidence-based treatments, including cognitive elements, with youth participants have also been shown to be effective, even when delivered by lay providers.

To explore whether the CETA approach could be provided in fewer sessions and with fewer elements, yet retain effectiveness, we designed a five-session intervention that is "front-loaded" with the elements that are most commonly used in evidence-based practices per distillation work [30], the empirical academic literature, and research studies conducted in LMIC with lay providers. Our working hypothesis is that these elements are the "strongest" mechanisms of action and, given past research in LMIC, are feasible elements for lay providers to deliver effectively. The study design is a non-inferiority trial and a trial of effectiveness by comparing a five-session, "front-loaded," Brief version of CETA to Standard CETA (8-12 sessions) and both versions to a wait-list control.

\section{Methods/design}

\section{Study aims}

This trial is designed to test two primary study hypotheses: (1) that a brief five-session version of CETA (Brief CETA) will provide similar effectiveness for reducing the severity of depression and post-traumatic stress (PTS) symptoms and improving daily functioning compared with the standard 8-12-session version of CETA (Standard CETA) and (2) that both Brief and Standard CETA will provide substantial impact for these same outcomes compared to a wait-list control condition. For both hypotheses, the main effect will be assessed 6 months post baseline.

Secondary aims include an exploration of implementation factors, including treatment fidelity, barriers and facilitators to the intervention implementation at the client and provider levels, and an exploration of unexpected additional effects of the program, both positive and negative. Planned secondary analyses include exploration of moderators of treatment impact by severity of disorders and comorbidity, examination of the impact of individual therapeutic components, and trends in symptom change between end of treatment and 6-month assessment for those in either Brief or Standard CETA.

\section{Study context}

This study is being conducted in Ukraine. Starting at the end of 2013 through early 2014, Ukraine experienced a period of rapidly increasing and widespread political discontent. In March 2014, Russia annexed Crimea in Eastern Ukraine, which began a series of violent events that continue today between pro-Russian separatists (supported by Russia) and Ukrainians supportive of the new government. These events have resulted in significant social and economic disruption and dislocation, with many individuals and families experiencing significant trauma and violence. This has included large numbers of individuals from across the country being conscripted into military service, hundreds of thousands of people being displaced from the eastern regions of Donetsk and Luhansk (the Donbass) and Crimea, and host communities being impacted by the influx of internally displaced persons. According to the Internal Displacement Monitoring Center [31], there are nearly 1.7 million internally displaced persons in Ukraine as of August 2015 with likely more who are displaced but not officially registered.

Between February and March of 2016, we conducted a brief qualitative study with internally displaced persons from the Donbass and military veterans living in two eastern oblasts, or regions, of Ukraine: Zaporizhia and Kharkiv. The aim of this formative study was to inform the adaptation of the CETA intervention to the Ukrainian context. The study sample included youth and adult internally displaced persons, veterans and their family members (wives and children), as well as community key informants and mental health professionals. We carried out free-list interviews, key informant interviews, and focus group discussions (FGDs) with a total of 226 participants. The qualitative study explored: participants' perceptions of significant problems faced by internally displaced persons and veterans; descriptions of local functioning behaviors; and experiences with accessing, and delivering, mental health services. 
Analysis allowed for the identification of psychosocial and mental health problems and symptoms of distress that were relevant to the study population. Both internally displaced persons and veterans described experiences of social isolation, difficulties with adaptation and (re-)integration, and conflicts with family members. Internally displaced persons also described missing home, fear, having a bad relationship with local residents/ lack of acceptance by the community, and conflicts in school among children. Veterans additionally named problems with substance use (alcohol and drug use), community members who are against them, feelings of being misunderstood by family members and the community, and aggression. For both groups, it was critical that mental health providers be trustworthy, accepting, and understanding of internally displaced persons and veterans' status and experiences, as well as competent and effective.

\section{Study design}

This study is conducted as a Hybrid Implementation-Effectiveness Type II [32] three-armed, single-blinded, randomized controlled trial in three cities in Ukraine. The study compares Brief CETA to Standard CETA and both Brief and Standard CETA to a wait-list control condition on our primary outcomes of depression, PTS symptoms, and daily functioning. A secondary aim is to evaluate implementation outcomes. The study design adheres to the Standard Protocol Items: Recommendations for Intervention Trials (SPIRIT) guidelines, including a SPIRIT Flow diagram, SPIRIT Schedule, and Checklist (Fig. 1 and Additional file 1).

\section{Participants and eligibility criteria}

Recruitment for this trial began in March 2017 and is ongoing. The first participant was enrolled in the trial on 8 March 2017. Participants in this trial are adults living in or near three Ukrainian cities-Kyiv, Kharkiv, and Zaporizhia-who meet the following inclusion criteria:

- Adults aged 18 years or older

- Being either an internally displaced person from Eastern Ukraine (the Donbass or Crimea); a veteran of the Anti-Terrorist Operations (ATO) in Ukraine (including adult family members); a volunteer in the "revolution of dignity" in the war in Eastern Ukraine, or in support of internally displaced persons or veterans from these conflicts; or any other non-military person affected by the conflict in Eastern Ukraine

- Elevated depression and/or PTS symptoms as defined by:

- A score of 7 or above on the locally validated depression scale (possible score range: $0-24$ ) or
- A score of 9 or above on the locally validated Post-Traumatic Stress Scale (possible score range: $0-39$ ) or

- A score of 7 or above on the locally validated depression scale and 9 or above on the locally validated Post-Traumatic Stress Scale

- Evidence of impaired daily functioning as assessed by a score of 4 or above on a locally validated scale of impaired functioning (possible score range: $0-32$ )

- Living in or around the three study cities for the duration of the study (at least 6 months) and able to regularly attend at least one of the locations where CETA is available

There are no restrictions on eligibility due to receiving other services. Participants who met the above criteria and who receive other health, mental health, social, behavioral, and any other type of services can be included.

Exclusion criteria include:

- An adult who is still a member of the armed forces (i.e., not a veteran)

- Acute suicidal or homicidal risk or active psychosis requiring immediate referral to psychiatric services

- Severe co-occurring medical problem that limits the ability to attend regular counseling sessions and/or requires immediate hospitalization

Assessors asked about suicide risk during the initial baseline assessment done on a tablet. If a possible participant responded "yes" to a single question about suicidal ideation in the past 2 weeks, they were asked four follow-up questions by the staff facilitating the baseline to gauge immediate risk. If someone answered positively to any of these follow-up questions, the assessor immediately called their supervisor, who provided clinical guidance by phone to assess for further risk, including past suicidal behavior. The supervisor was able to consult with clinical supervisors if they were unsure of the person's level of risk. If the person was judged to be at immediate risk (i.e., past attempt and current ideation, a plan, and the means to carry out that plan) they were referred to a psychiatrist (who saw them within 24 hours), responded to by a local emergency psychiatric service, or driven directly to a hospital and were not randomized into a treatment condition. However, it was possible for a potential participant to be "cleared" by a psychiatrist and subsequently randomized into the trial. All clients were who were determined to have some risk, but not immediate risk, were asked to sign a safety contract and a safety plan was made to insure the person was checked on regularly and knew what to do and who to call if they started having suicidal thoughts. Any individual who could not respond to questions or presented with a severe illness was referred to a 
SPIRIT Figure - Schedule of enrolment, interventions, and assessments

\begin{tabular}{|c|c|c|c|c|c|c|c|c|c|c|c|c|c|c|c|c|c|c|}
\hline & & & & & & & rUD & $Y P$ & ERI & OD & & & & & & & & \\
\hline & Enrolment & Allocation & & & Int & $\begin{array}{l}\text { terv } \\
\text { e in }\end{array}$ & we & $\begin{array}{l}\text { ion } \\
\text { eks }\end{array}$ & & & & $\begin{array}{r}P \\
(w=)\end{array}$ & $\begin{array}{l}\text { st-i } \\
\text { wee }\end{array}$ & $\begin{array}{l}\text { iterv } \\
; \mathrm{m}\end{array}$ & $\begin{array}{l}\text { entio } \\
=\text { mo }\end{array}$ & hth) & & $\begin{array}{l}\text { Close- } \\
\text { out }\end{array}$ \\
\hline MIMEPUINI & $\begin{array}{l}1 \text { Mar } 2017 \text { - } \\
31 \text { May } 2018\end{array}$ & 0 & 1 & 2 & 3 & 4 & 5 & 6 & 7 & $8+$ & $\begin{array}{l}2 \\
w\end{array}$ & $\begin{array}{l}1 \\
m\end{array}$ & $\begin{array}{l}2 \\
m\end{array}$ & $\begin{array}{l}3 \\
m\end{array}$ & $\begin{array}{l}4 \\
m\end{array}$ & $\begin{array}{l}5 \\
m\end{array}$ & $\begin{array}{l}6 \\
m\end{array}$ & $\begin{array}{c}1 \text { Jun } \\
2018- \\
31 \text { Dec } \\
2018\end{array}$ \\
\hline ENROLMENT: & & & & & & & & & & & & & & & & & & \\
\hline Eligibility screen & $X$ & & & & & & & & & & & & & & & & & \\
\hline Informed consent & $\mathrm{X}$ & & & & & & & & & & & & & & & & & \\
\hline Allocation & & $x$ & & & & & & & & & & & & & & & & \\
\hline INTERVENTIONS:" & & & & & & & & & & & & & & & & & & \\
\hline Brief CETA & & & $x$ & $x$ & $x$ & $x$ & $x$ & & & & & & & & & & & \\
\hline Standard CETA & & & $x$ & $x$ & $x$ & $x$ & $x$ & $x$ & $x$ & $x$ & & & & & & & & \\
\hline 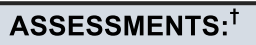 & & & & & & & & & & & & & & & & & & \\
\hline Demographics & & $X$ & & & & & & & & & & & & & & & & \\
\hline $\begin{array}{l}\text { Life events } \\
\text { checklist }\end{array}$ & & $x$ & & & & & & & & & & & & & & & & \\
\hline $\begin{array}{l}\text { Depression }{ }^{* *} \\
\text { (IDSS) }\end{array}$ & & $x$ & & & & & & & & & $X$ & $X$ & $x$ & $x$ & $X$ & $x$ & $x$ & \\
\hline $\begin{array}{l}\text { Post-traumatic }{ }^{* *} \\
\text { stress (GPTSS) }\end{array}$ & & $x$ & & & & & & & & & $x$ & $\mathrm{x}$ & $\mathrm{x}$ & $x$ & $X$ & $x$ & $x$ & \\
\hline $\begin{array}{l}\text { Impaired function }{ }^{* *} \\
\text { (WHODAS 2.0; } \\
\text { local items) }\end{array}$ & & $x$ & & & & & & & & & $\mathrm{X}$ & $\mathrm{x}$ & $\mathrm{x}$ & $x$ & $x$ & $x$ & $\mathrm{x}$ & \\
\hline $\begin{array}{l}\text { Anxiety* }{ }^{*}(H S C L \\
\text { sub-scale) }\end{array}$ & & $x$ & & & & & & & & & $x$ & $X$ & $x$ & $X$ & $X$ & $X$ & $x$ & \\
\hline $\begin{array}{l}\text { Alcohol use* } \\
\text { (ASSIST 3.0) }\end{array}$ & & $\mathrm{x}$ & & & & & & & & & $x$ & $x$ & $x$ & $x$ & $X$ & $x$ & $x$ & \\
\hline $\begin{array}{l}\text { Drug use } \\
\text { (ASSIST 3.0) }\end{array}$ & & & & & & & & & & & $x$ & $x$ & $x$ & $x$ & $X$ & $x$ & $x$ & \\
\hline Suicide ideation & & $x$ & & & & & & & & & $x$ & $\mathrm{x}$ & $x$ & $x$ & $x$ & $x$ & $\mathrm{x}$ & \\
\hline $\begin{array}{l}\text { Ideation about } \\
\text { harming others }\end{array}$ & & $x$ & & & & & & & & & $X$ & $\mathrm{x}$ & $x$ & $x$ & $x$ & $x$ & $x$ & \\
\hline $\begin{array}{l}\text { Use of alternate } \\
\text { psychiatric services }\end{array}$ & & & & & & & & & & & $\mathrm{X}$ & $x$ & $x$ & $x$ & $x$ & $x$ & $x$ & \\
\hline $\begin{array}{l}\text { Implementation - } \\
\text { participants }\end{array}$ & & & & & & & & & & & & $x$ & & & & & & \\
\hline $\begin{array}{l}\text { Implementation - } \\
\text { providers }\end{array}$ & & & & & & & & & & & & & & & & $x$ & & \\
\hline
\end{tabular}

** Denotes primary outcome.

* Denotes secondary outcome.

\# Participants assigned to wait-control arm of the trial receive either Brief or Standard CETA after their wait period.

$\dagger$ Assessments are shortened versions of these original scales indicated in parentheses. Analysis based on tem response theory was used to shorten the original scales.

Fig. 1 Standard Protocol Items: Recommendations for Interventional Trials (SPIRIT) Figure; schedule of enrollment, interventions, and assessments

psychiatrist or physician and not included in the trial. Homicidal risk was not asked about explicitly, but if it came up during the baseline assessment or during any additional suicide safety procedures, the staff contacted a supervisor to create and sign a safety contract and made a referral to a psychiatrist to be seen within 24 hours.

\section{Recruitment and baseline assessment}

The participant flow is shown in Fig. 2. Both internally displaced persons and veterans are recruited through local service providers including government and non-governmental organizations (NGOs), through referral by study counselors from their existing client base, and through self-referral. Staff at the local NGOs were provided with a locally validated brief instrument that assessed symptoms of depression, anxiety, and PTS. This screening instrument is based on a previous validity study and Item Response Theory (IRT) analysis and includes the same symptom questions as the main study instrument, but does not ask about suicidal ideation or 


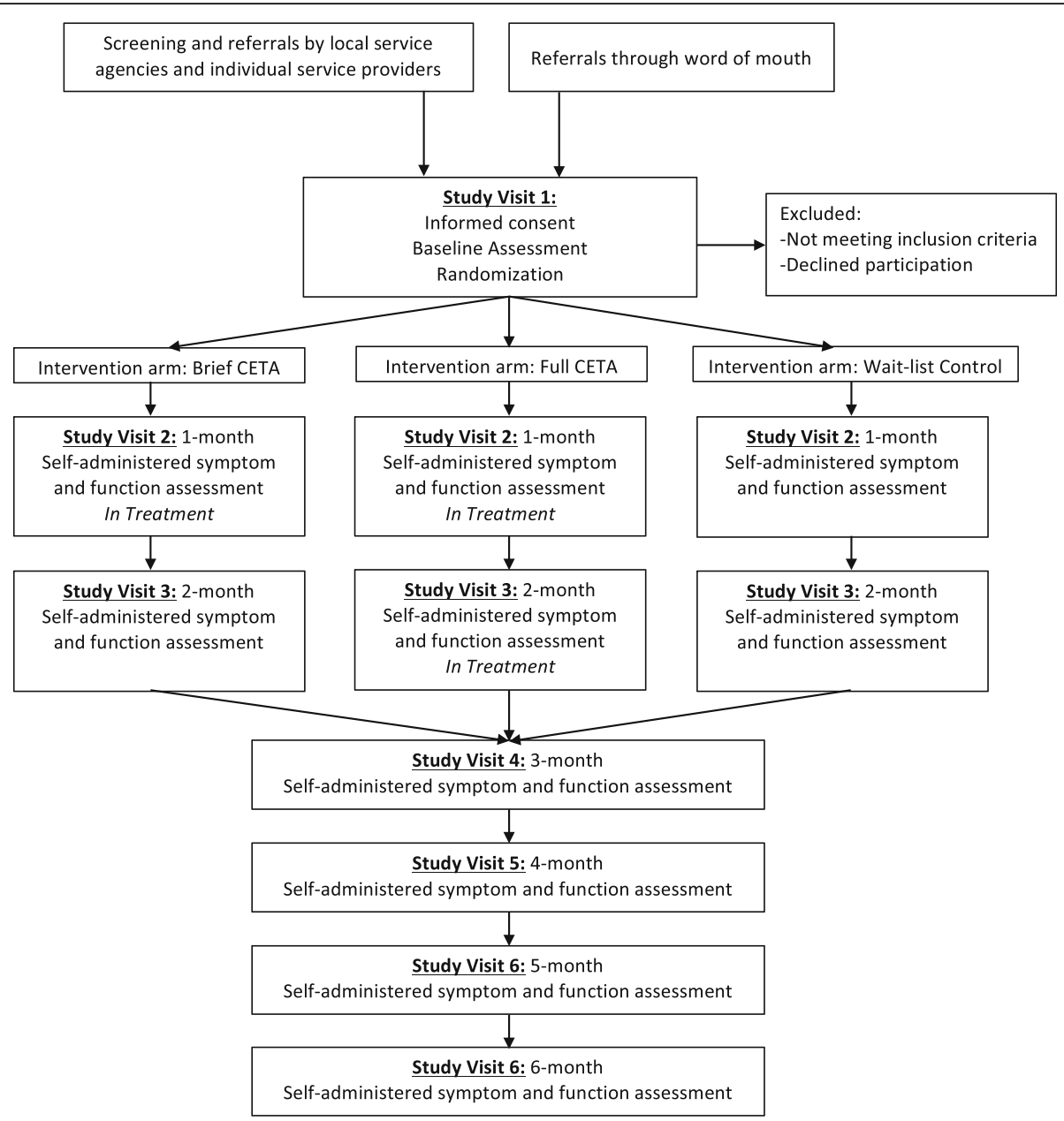

Fig. 2 Study protocol flow

impaired functioning. As part of their regular services, the referring organizations use this instrument to assess their clientele for need of mental health services. When the instrument indicates depression and/or PTS symptoms, the person is informed about the study and asked if they agree to be contacted by a member of the study team.

Once a person has been identified as having elevated depression and/or PTS symptoms and agrees to be contacted, a study staff member sets a time to meet in person, complete consent procedures and conduct the baseline assessment to ensure trial eligibility. Both informed consent and the baseline assessment are self-administered on an electronic tablet. The study staff is present to facilitate the process, troubleshoot problems with the tablet and answer any questions, but will allow the potential participant to respond to questions in private. Consent is via a secure data collection/management software program (CommCare) [33] on a password-protected, handheld tablet. If the person does not consent to participate in the trial, the baseline assessment is not administered. If the person consents to the trial and completes the baseline, the person is immediately notified if they are eligible and whether they will receive treatment immediately or are asked to wait. If randomized to receive CETA, the participant is not told if they have been allocated to Brief or Standard CETA. Individuals who are not eligible for the study (either by not meeting the inclusion criteria or meeting one or more of the exclusion criteria) are thanked for their time and given a list of service organizations in their city.

\section{Baseline assessment}

The baseline assessment consists of demographic information and scales to measure our primary outcomes: symptoms of depression, PTS, and impaired functioning; and secondary outcomes: alcohol use and anxiety symptoms. Briefly, based on our initial qualitative work, we identified, adapted, and tested appropriate mental health scales, including the International Depression Symptom Scale (IDSS) [34], the Global Post-traumatic Stress 
Symptom Scale (GPTSS) [35], the Anxiety sub-scale of the Hopkins Symptom Checklist (HSCL-A), and the Alcohol Smoking and Substance Involvement Screening Test (ASSIST 3.0) [36, 37]. The IDSS and GPTSS were developed based on presentation of depression and PTS globally. For anxiety we used the HSCL-A [38] given its demonstrated validity in diverse populations, including those affected by political conflict [39-42]. For alcohol use, we used the alcohol section of the ASSIST 3.0, developed by the World Health Organization as a cross-cultural screener for substance use problems. It consists of eight questions, focused on frequency, dependence, and functional consequences of use. To assess impaired functioning (primary outcome), we used the World Health Organization's Disability Adjustment Schedule 2.0 (WHODAS 2.0) 12-item version and a set of local function items. The WHODAS 2.0 is a generic instrument developed to provide a standardized measurement of functional impairment across cultures [43]. The set of local function items measures the degree of difficulty people experience when performing activities of daily living that are salient to the local population [44].

All of these outcome measures were translated into Russian and the full battery was tested during an instrument validation study among a sample of $N=153$ internally displaced persons and veterans living in or around the urban areas of Zaporizhia and Kyiv [45]. The validation analyses involved testing reliability and validity, including criterion validity by comparing scores on the IDSS, GPSSS, HSCL-A, and ASSIST to local psychiatrist diagnosis using the Structured Clinical Interview for DSM-IV-Research Version (SCID-IV-RV) [46]. To further refine and shorten the measure, we performed a secondary data analysis of the validation data using IRT. The original assessment battery tested during the validation study had a total of 153 items, and IRT analysis resulted in a 37-item assessment that reliably and validly measures depression, PTS, impaired functioning, alcohol use and generalized anxiety (Table 1). Eligibility cutoff scores were developed by comparing average scores on each of the shortened measures to Structured Clinical Interview for DSM (SCID) diagnoses and generating scores that maximized both sensitivity and specificity for a given outcome [47]. These measures are administered at baseline for screening purposes and on a monthly basis and serve as the main study outcome (Table 2).

\section{Study setting}

The study is being conducted by researchers at the Johns Hopkins Bloomberg School of Public Health in collaboration with researchers from the National University of Kyiv-Mohyla University. The CETA treatment sessions take place at various locations in the three study sites in Ukraine-the urban areas of Kyiv, Kharkiv, and Zaporhizhia. Kyiv, the capital, is the largest city in Ukraine with a population of approximately 2.8 million [48] and is located in the north central part of the country. It has a large number of internally displaced persons and veterans. Kharkiv is the second largest city, with around 1.5 million residents, [48] and is located close to the Russian border in east Ukraine. It was the first capital city for Ukraine under the Soviet Union and is a prominent regional center of scientific and medical education. Zaporizhia has a population of around 800,000 is an important manufacturing and industrial hub in

Table 1 Common Elements Treatment Approach (CETA) trial aim 1 evaluation measures

\begin{tabular}{|c|c|c|c|c|c|}
\hline Measures & Description of measure & Number of items & Cronbach's alpha & Test-retest reliability & Area under the curve $e^{a}$ \\
\hline \multicolumn{6}{|l|}{ Primary outcomes } \\
\hline Depression symptoms & $\begin{array}{l}\text { Items taken from the International } \\
\text { Depression Symptom Scale (IDSS), a } \\
\text { measurement instrument designed } \\
\text { to represent global presentations of } \\
\text { depression [27] }\end{array}$ & 8 & $a=0.89$ & Rho $=0.87$ & $A \cup C=0.78$ \\
\hline $\begin{array}{l}\text { Post-traumatic stress } \\
\text { symptoms }\end{array}$ & $\begin{array}{l}\text { Items taken from the Global Post } \\
\text { Traumatic Stress Symptom Scale } \\
\text { (GPTSSS) [28] }\end{array}$ & 12 & $a=0.91$ & Rho $=0.87$ & $A \cup C=0.68$ \\
\hline Impaired function & $\begin{array}{l}\text { A combination of items from the } \\
\text { WHODAS [36] and a locally derived } \\
\text { function scale based on qualitative } \\
\text { data [37] }\end{array}$ & 8 & $a=0.88$ & Rho $=0.94$ & $A \cup C=0.72$ \\
\hline \multicolumn{6}{|l|}{ Secondary outcomes } \\
\hline Anxiety symptoms & $\begin{array}{l}\text { Items taken from the Hopkins } \\
\text { Symptom Checklist Anxiety } \\
\text { sub-scale }\end{array}$ & 4 & $a=0.82$ & Rho $=0.80$ & $-{ }^{b}$ \\
\hline Alcohol use & $\begin{array}{l}\text { Items taken from the ASSIST alcohol } \\
\text { sub-scale [29] }\end{array}$ & 2 & - & Rho $=0.80$ & $A \cup C=0.90$ \\
\hline
\end{tabular}

ahen compared to corresponding diagnoses provided by Ukrainian psychiatrists using the Structured Clinical Interview for DSM-IV

${ }^{b}$ Insufficient sample size to calculate AUC due to only six participants diagnosed with generalized anxiety disorder 
Table 2 Items in each Common Elements Treatment Approach (CETA) trial aim 1 evaluation measure

\begin{tabular}{|c|c|c|c|c|c|}
\hline & Depression & Posttraumatic Stress & Impaired Function & Anxiety & Alcohol Use \\
\hline 1 & Feeling sad & Feeling sad & $\begin{array}{l}\text { Taking care of your household } \\
\text { responsibilities? }\end{array}$ & $\begin{array}{l}\text { Frequently trembling or } \\
\text { shaking }\end{array}$ & $\begin{array}{l}\text { In the past week, how often } \\
\text { have you used alcoholic } \\
\text { beverages? }\end{array}$ \\
\hline 2 & $\begin{array}{l}\text { Feeling no interest in things/less } \\
\text { interest in daily activities }\end{array}$ & $\begin{array}{l}\text { Feeling no interest in } \\
\text { things/less interest in daily } \\
\text { activities }\end{array}$ & $\begin{array}{l}\text { Dealing with people you do } \\
\text { not know? }\end{array}$ & $\begin{array}{l}\text { Nervousness or shakiness } \\
\text { inside }\end{array}$ & $\begin{array}{l}\text { In the past week, how often } \\
\text { have you had a strong desire to } \\
\text { use alcoholic beverages? }\end{array}$ \\
\hline 3 & Crying easily & Crying easily & Your day-to-day (daily) work & Feeling tense or keyed up & \\
\hline 4 & Feeling lonely; Feeling isolated & Feeling lonely; Feeling isolated & Helping your family & Spells of terror or panic & \\
\hline 5 & $\begin{array}{l}\text { Feeling tired, low in energy or } \\
\text { slowed down }\end{array}$ & $\begin{array}{l}\text { Feeling tired, low in energy or } \\
\text { slowed down }\end{array}$ & $\begin{array}{l}\text { Joining in community } \\
\text { activities and cultural events } \\
\text { (e.g. going on walks, going to } \\
\text { museums, going to the theater }\end{array}$ & & \\
\hline 6 & $\begin{array}{l}\text { Feeling of being trapped or } \\
\text { caught }\end{array}$ & $\begin{array}{l}\text { Feeling of being trapped or } \\
\text { caught }\end{array}$ & Helping others & & \\
\hline 7 & Feelings of worthlessness & $\begin{array}{l}\text { Feeling emotionally upset } \\
\text { when something reminded me } \\
\text { of the stressful event }\end{array}$ & $\begin{array}{l}\text { Doing hobbies (e.g. making } \\
\text { crafts, sewing, embroidery, } \\
\text { hunting, fishing, reading, } \\
\text { games) }\end{array}$ & & \\
\hline 8 & $\begin{array}{l}\text { Moving or speaking so slowly } \\
\text { or so fast that others have } \\
\text { noticed }\end{array}$ & Feeling that no one understands & Conversing with others (U) & & \\
\hline 9 & & $\begin{array}{l}\text { Avoiding thoughts/memories } \\
\text { related to the past stressful } \\
\text { event }\end{array}$ & & & \\
\hline 10 & & $\begin{array}{l}\text { Avoiding activities or } \\
\text { situations that are in generally } \\
\text { safe that remind me of the past } \\
\text { stressful event }\end{array}$ & & & \\
\hline 11 & & $\begin{array}{l}\text { Feeling a loss of your sense of } \\
\text { self }\end{array}$ & & & \\
\hline 12 & & $\begin{array}{l}\text { Frequently trembling or } \\
\text { shaking }\end{array}$ & & & \\
\hline
\end{tabular}

*Outcomes in box indicate primary outcomes

Southeastern Ukraine [48]. Neither Zaporizhia nor Kharkiv oblasts (regions) were occupied by Russian forces, but both border Donetsk and Luhansk and contain significant numbers of internally displaced persons and ATO veterans, as does Kyiv. Meeting locations vary, and are based on where participants feel most comfortable. Locations include private offices, veterans' centers, internally displaced person service centers, and individual's homes. A total of 39 Ukrainian psychologists, social workers, and veterans completed a training in CETA using the apprenticeship model [49]. Two authors (LKM, SD) were responsible for the training of the CETA providers and supervisors.

\section{Interventions}

\section{CETA intervention}

As described in the introduction section, the Common Elements Treatment Approach (CETA) is a modular, flexible, transdiagnostic approach developed by our team specifically for use by providers who need not be formally trained mental health professionals. The number of elements was purposefully limited to help with training, mastery, and scalability. Elements are cognitivebehavioral and include: Engagement, Introduction, Thinking in a different way 1 and 2 (i.e., cognitive processing), Talking about difficult memories (i.e., imaginal exposure), Relaxation, Getting Active (i.e., Behavioral Activation), Problem solving, and a Cognitive-behavioral approach to substance abuse (see Table 3). The implementation of CETA elements can vary on: (1) selection of elements, (2) order of elements, and (3) dose of elements depending on symptom presentation. Providers are taught to use an assessment tool and what the client "does and says" to determine the primary problem(s). Elements are chosen based on the primary problem(s) with orders that mimic evidence-based treatments. For example, if someone comes in with primarily trauma-related problems such as nightmares, avoiding trauma-related stimuli, but also other problems (e.g., sadness, not engaging in pleasurable activities, relationship issues), an initial choice and order of elements would mimic trauma cognitive-behavioral treatments of: Engagement/Introduction, Thinking in a different way 1 , Talking about difficult memories (usually two to five 1-hour sessions), and Thinking in a different way 2 (usually one to two 1-hour sessions). If depressive symptoms are still present after these elements are delivered, or if strong depressive symptoms are interfering, the element of Getting Active (i.e., Behavioral Activation) may be added. As developed, CETA can provide individualized targeting of treatment with the number of sessions delivered dependent on reduction in symptoms. Further details can be found in a description paper [21] and within the two clinical trials published to date on CETA $[26,28]$.

We developed a Brief CETA version with five sessions to address concerns around scalability and time limitations 
Table 3 Elements of Common Elements Treatment Approach (CETA)

\begin{tabular}{|c|c|c|}
\hline Element & $\begin{array}{l}\text { Simplified name } \\
\text { (used in training) }\end{array}$ & Description \\
\hline Psychoeducation and engagement & Introduction & $\begin{array}{l}\text { - Focus on obstacles to engagement } \\
\text { - Linking program to assisting with } \\
\text { client's problems } \\
\text { - Includes family when appropriate. } \\
\text { Program information (duration, } \\
\text { content, expectations) } \\
\text { - Normalization/validation of current } \\
\text { symptoms/problems }\end{array}$ \\
\hline Anxiety management strategies & Relaxation & $\begin{array}{l}\text { - Strategies to improve physiological } \\
\text { stress } \\
\text { - Examples include: deep breathing, } \\
\text { meditation, muscle relaxation, and } \\
\text { imagery. Others added by local cultures }\end{array}$ \\
\hline Behavioral Activation & Getting Active (GA) & $\begin{array}{l}\text { - Identifying and engaging in } \\
\text { pleasurable, mood-boosting, or } \\
\text { efficacy-increasing activities }\end{array}$ \\
\hline Cognitive coping/Restructuring & $\begin{array}{l}\text { Thinking in a Different Way (TDW) - } \\
\text { Part I and Part II } \\
\text { TDW1 and TDW2 }\end{array}$ & $\begin{array}{l}\text { - Understand association between } \\
\text { thoughts, feelings, and behavior } \\
\text { - Learn to restructure thinking to be } \\
\text { more accurate and/or helpful }\end{array}$ \\
\hline Imaginal Gradual Exposure & Talking about trauma memories (TDM) & $\begin{array}{l}\text { - Facing feared and avoided memories } \\
\text { in detail } \\
\text { - Gradual desensitization/exposure }\end{array}$ \\
\hline $\begin{array}{l}\text { Suicide/homicide/danger } \\
\text { assessment and planning }\end{array}$ & Safety & $\begin{array}{l}\text { - Assessing client risk for suicide, } \\
\text { homicide, and domestic violence } \\
\text { - Developing a focused plan with the } \\
\text { client and client's family (when } \\
\text { appropriate) } \\
\text { - Additional referral/reporting when } \\
\text { needed }\end{array}$ \\
\hline $\begin{array}{l}\text { Cognitive-behavioral therapy } \\
\text { (CBT) for substance use and } \\
\text { relapse prevention }\end{array}$ & Substance use element (SU) & $\begin{array}{l}\text { - Utilizes concepts of Motivational } \\
\text { Interviewing to get client buy-in to } \\
\text { change substance use/abuse behavior }\end{array}$ \\
\hline
\end{tabular}

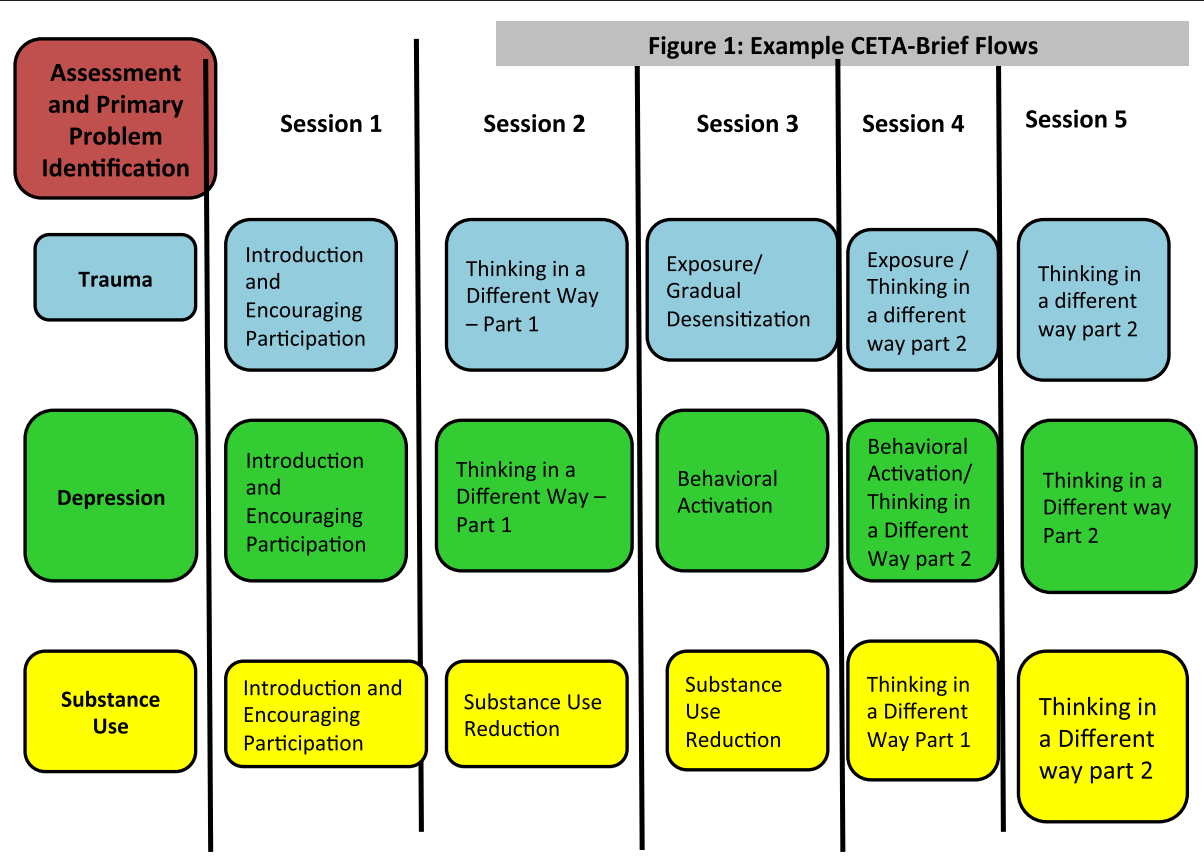

Fig. 3 Example Common Elements Treatment Approach (CETA)-Brief flows 
within low-resource settings and certain contexts. All Brief CETA flows include one cognitive element and one behavioral element, and an introduction session. (See Fig. 3). Counselors, supervisors and trainers first agree on a primary presenting problem (trauma, depression, anxiety, or substance use). Each problem area has a set flow of certain elements based on evidence-based treatments for these primary areas. For example, the two most commonly used evidence-based elements in treatments for trauma are Cognitive coping/restructuring and Gradual desensitization or exposure [50, 51]. For depression, most evidence-based treatments include Cognitive coping/Restructuring and/or Behavioral Activation, thus the Brief CETA flow contains only these. Since Cognitive coping/Restructuring is present in all flows, this may be used to target multiple or varying symptomatology (e.g., depression thoughts, unhelpful thoughts related to the traumatic event).

Since randomization occurs after the fourth session, all participants begin with one of the Brief CETA five-session flows. Those randomized to Standard CETA receive additional sessions based on the Standard CETA protocol including evaluating: (1) client report of symptoms, (2) what the client does and says, and (3) supervision. A provider needs to provide at least three additional sessions, and more could be provided as needed. Completion of Standard CETA is based on decreased symptoms, improvement report by client, and supervisor approval. This Standard CETA arm models a clinical "stepped care" approach wherein a client would be offered a "front-loaded Brief CETA" first, and then if symptoms persist, treatment can be augmented with additional sessions and/or elements [52].

\section{Control condition}

Participants assigned to the wait-list control condition are asked to wait for approximately 6 months before being offered the Stanard CETA intervention. Wait-list control participants undergo monthly monitoring after enrollment in the study. Monitoring is done via in-person meeting or, if necessary, a phone call, to ensure ongoing connection with the study; to obtain information on current mental health status including high-risk symptoms (i.e., suicidality) that would require immediate assistance; and to record any other services they receive. Control participants are administered the same symptom items and impaired function scale as the baseline assessment as CETA participants. Study monitoring and evaluation staff are responsible for these monthly assessments. During the wait period, participants are free to use other social and mental health services. A single item on the assessment asks whether the participant has obtained other services, and if so, which ones. Study monitoring and evaluation staff provides participants with a list of other psychosocial services in their respective cities during the baseline assessment visit.

\section{CETA providers and training}

Thirty-nine Ukrainian individuals were initially trained, with 34 continuing onto the study (seven men; 32 women). They hold a range of positions including social worker (9), psychologist (12), volunteers (e.g., with psychological crisis services) (5), physician (1), program manager (4), teacher/lecturer (2), and lawyer (1). A psychologist in Ukraine will have received approximately 2 years of post-university training in psychological theory but without applied clinical experience. Social workers are usually based within social services and are not trained to provide psychological treatments. In the training we found that most trainees did not have experience providing talk therapy directly to clients. Most were educated in Gestalt Theory and had little background in CBT.

CETA training and supervision followed the Apprenticeship Model (see Murray et al. [49] for details). Ukrainian providers $(N=39)$ received a 10 -day training in CETA (May 2016), and then subsequently participated in small practice groups led by local supervisors (July 2016 to February 2017). They were taught all components, as well as how to make decisions about selection, sequencing, and dosing (i.e., tailoring to the individual participant) based on three sources of information: (1) results from certain items on the validated study instrument, (2) client observations and statements in the assessment and early sessions, and (3) discussion with their supervisor.

Initially, eight supervisors were included in supervision training. Some were not able to commit to the time required for supervision, leaving five supervisors for the trial (four women). Two are psychologists, one of whom is also a professor of psychology at the National University of Kyiv-Mohyla Academy. One is a social worker, one is a project coordinator, and another is a supervisor for a substance abuse foundation program. The supervisors received an additional 8 hours during the initial training to review supervision skills, although at least 4 hours of this time ended up being logistical problem solving. From April 2016, supervisors are in weekly communication with a CETA trainer to review the agendas, cases, implementation of elements, decisionmaking on CETA, weekly symptom monitoring scores, what happens each session, and supervision skills [21]. These calls are usually through Skype and last approximately 2 hours each.

\section{Intervention fidelity}

Fidelity to CETA is tracked through a three-tiered system. First, CETA providers complete session notes and report to their supervisors each week, providing self-report of fidelity. These session notes include: date, session number, elements done in session and for what 
duration, weekly monitoring scores, "yes/no" to suicide/ homicide risk questions, details about what was actually done in session (qualitatively), and the plan for the next session. Second, informed by this information and additional queries and discussion between the counselor and supervisor (e.g., "tell me exactly how you introduced the thinking in a different way triangle to your client."), supervisors document this same information in an Excel sheet. Supervisors also complete their own ratings evaluating providers' fidelity, including: (1) adherence (i.e., how well the counselor followed the elements stepsheets), (2) review of homework, (3) engagement, (4) explaining the what and why of the element used, (5) competency (i.e., overall skill at teaching the element), (6) assigning homework, and (7) an overall, global rating of the counselor for that session. Ratings for each fidelity domain are done on a 1-5-point scale with 1 being the lowest rating and 5 being the highest rating. Third, each week the trainer reviews the local supervisors' spreadsheet documentation and ratings (via a shared Excel file). Trainers document the time supervisors spent in supervision, what was covered, and the time spent between a supervisor and trainer. The trainer then rates the supervisors' ability on: (1) ability to notice mistakes, (2) responding to counselor questions or mistakes with correct direction, (3) ability to objectively report to the trainer, (4) the number of counselors who need to change the plan per the trainer, and (5) an overall rating. If a counselor did not adhere to the plan or did not implement the element following the steps, they repeat the element or the steps they missed in the following session. No counselor is allowed to have a session until they have discussed the previous session with the supervisor, and the supervisor has discussed it with the trainer. Any safety concerns (e.g., suicidal) are documented in a safety log, and reported immediately to the CETA trainers and study investigators. Each case is addressed individually based on clinical needs and may include daily safety checks, safety contracts, and possibly referral to a psychiatrist. Fidelity documentation also tracks any changes to the flows throughout treatment, why the change was made, and by whom. This will allow us to look at the correlation and learning time of decision-making.

\section{Outcome and study measures}

The study's primary aim is to determine intervention effectiveness comparing Standard to Brief CETA, and both to a control group. For this aim, the primary outcomes are symptoms of depression, PTS, and impaired functioning (Tables 1 and 2) measured on a monthly basis for 6 months. Secondary outcomes include symptoms of anxiety and problematic alcohol use, also measured on a monthly basis for 6 months (see Tables 1 and 2 and Fig. 1). Outcome assessments for our primary aim of determining intervention effectiveness are done at baseline prior to randomization, and on a monthly basis for approximately 6 months.

All data is collected via self-administered digital survey on an electronic tablet device using CommCare [33]. Baseline assessments are done at a central location or at another private location convenient to the participants. Monthly assessments consist of the same symptom and functioning items as the baseline assessment (Table 2). For the control group, the study staff sets up monthly assessment appointments to be done at a central location and are self-administered on CommCare with the same symptom and functioning items as the baseline assessment (Table 2).

For the treatment groups, the monthly assessment is incorporated into a clinical monitoring form (Table 2). The clinical monitoring form includes the impaired function items every fourth visit (i.e., monthly) rather than on a weekly basis. The clinical monitoring form is self-administered on CommCare at the beginning of each CETA session and helps guide the course of treatment. After treatment ends, participants continue to be monitored monthly with the same assessment (Table 2) via in-person visit (or phone call if in-person is not possible) by the study team. At the last assessment ( 6 months post treatment), some additional demographic- and alcohol-related questions are administered to measure potential pre-post change on these characteristics.

A second aim of the study is to explore the implementation of CETA from the perspective of consumers and providers in the Ukrainian context, using a Hybrid Type II effectiveness-implementation design. To measure implementation factors, including barriers and facilitators to implementation, we use scales that cover the major implementation science domains of Adoption, Acceptability, Appropriateness, Feasibility, and Accessibility/Reach. These scales were previously tested among a sample of Ukrainian consumers and providers and found to be reliable and valid (Haroz et al: Measuring implementation in global mental health: an example from eastern Ukraine, under review). For consumers, these scales are administered via CommCare to Brief and Standard CETA participants at their second post-treatment monitoring assessment. Qualitative interviews with CETA participants $(N=60)$ are also conducted at this time. The qualitative interviews, using free listing, ask CETA participants about changes that they experienced during treatment, because of treatment, and what they would want to change about CETA. For CETA providers (counselors and supervisors), the implementation scales include additional constructs related to organizational leadership and capacity for sustainability, and will be administered near the end of the trial. Providers also participate in focus groups after 6 months of providing CETA services in order to discuss what they like, do not like, and what they would change about implementation of CETA. 


\section{Randomization, concealment, un-blinding}

All eligible and consented adults are randomly allocated by a computer-generated randomization algorithm to either a wait-list control condition, the Brief CETA model, or the Standard CETA model. The unit of randomization is the individual. Block randomization is used to ensure equal distribution of treatment and control participants across providers. Within a provider, we randomly allocate 20 participants using a 1:2:2 (control:Brief:Standard) allocation ratio. Participants, counselors, study staff, and all clinical supervisors are blinded to study group allocation between Brief and Standard CETA conditions. This is done in an effort to ensure that all participants across both Brief and Standard CETA conditions will receive comparable treatment (see section "Concealment" below).

Study group allocation, either treatment or control only, is revealed to eligible participants immediately following the baseline assessment. If assigned to begin treatment right away, the study staff assigns a participant to a counselor based on availability and location of treatment. Counselors set up an initial meeting with the participant within 10 days of the baseline assessment. If assigned to control, the study staff set up a study assessment visit approximately 30 days after the completed baseline assessment.

\section{Concealment}

At the initiation of treatment, all CETA providers, supervisors and trainers and participants are blind to whether a participant is randomized to Brief or Standard CETA. The informed consent describes the possibility of Brief vs. Standard to the participant. At the end of the fourth treatment session, the counselor reads a script to the participant reminding them that the next session may be the last session. Following completion of the fourth treatment session, the study staff team notifies the Johns Hopkins University (JHU) research team via email about the need to un-blind the Brief or Standard status. Only the study director and two analysts on the data monitoring team have access to the password-protected database linking study ID number to treatment status. Once an email request for un-blinding is received, the study director checks the randomized assignment and sends an email back to the research team, the supervisor, and the trainer with the participant status (Brief or Standard). The clinical supervisor then contacts the counselor to un-blind. This whole process is done within 24 hours of completion of the fourth treatment session so as to allow planning between counselor and supervisor for either ending or continuing treatment. The participant does not find out about Brief vs. Standard status until they arrive at the fifth session. If the participant is assigned to Brief but still interested in services, the counselor may give them a list of local services but may not continue seeing the client. The client is also prohibited from receiving CETA from another counselor while the trial is ongoing.

\section{Sample size}

All sample size and power calculations are based on longitudinal data collected monthly for controls and at every fourth visit for the CETA clients groups over the course of 6 months. (seven measurement time points). For our primary hypothesis, that Brief and Standard CETA would result in similar changes in symptoms of depression, PTS and impaired functioning, we used a standard deviation (SD) of 7.0 for depression symptoms at baseline (based on previous studies among trauma-affected populations) [26, 28]. Under the assumption that differences in outcomes that are less than one third of the standard deviation are considered not practically meaningful $[53,54]$, a non-inferiority margin of $\Delta<3$ was selected. The sample size that would yield a power of $0.80(\beta=0.20)$ or greater in testing at $\alpha=0.05$ level of significance, with an assumed SD of 7.0 and a non-inferiority limit of $d=2.4$ is $N=178$ (or $n=89$ in each CETA group). For aim 2, the sample size that would yield a power of $0.80(\beta=0.20)$ or greater in testing at $\alpha=0.025$ level of significance (adjusted for multiple comparisons), to detect a medium effect $(f=0.25)$ for the direct comparison of either Brief or Standard CETA to wait-list control would be $n=45$ in each group. As the sample size needed for aim 1 is the larger of the two calculations, we will use it as the basis for the total study sample planning $(n=223$ across the three arms; 89 in Brief CETA; 89 in Standard CETA; 45 in the control group). Accounting for 30\% attrition, a total of $N=294$ participants are needed for the randomised controlled trial (RCT).

For the implementation portion of the study, all study participants in both CETA arms are interviewed using a quantitative instrument (Consumer dissemination and implementation (D\&I) Instrument) but only the first 30 completers in each CETA arm receive the qualitative interview. All CETA providers (approximately 40) are interviewed with a quantitative instrument (Provider D\&I Instrument) and asked to participate in the FGDs.

\section{Data analyses \\ Primary analyses}

Study hypotheses will be tested using an "intent-to-treat" model. The primary and secondary outcomes of interest will be mental health symptoms and functioning measured monthly for 6 months for all groups. Given the longitudinal nature of the data, we will use mixed-effects regression models to estimate average treatment effects while accounting for shared variance of repeated measures within participant. Treatment effects will be measured based on within-person change in mean scores on the symptom and function measures. 
Statistical significance will be set at $p<0.05$. We will use Cohen's $d$ effect sizes to express the magnitude of the difference between treatment groups. Missing data will be explored as to the mechanism of missingness. If appropriate to assume missing completely at random or missing at random, we will use multiple imputation with chained equations to impute all missing data, including outcomes for those lost to follow-up [55, 56].

Analysis for secondary aims Analysis of the implementation data will include examination of distribution of scale-level scores represented as average responses for all items in each scale. Individual-level items with average scores lower than the average score on the scale will be considered possible barriers and used to identify program implementation areas that could be improved. Finally, we will examine whether more positive experiences of implementation, as measured by higher average scores on each implementation scale, is associated with different treatment conditions, client outcomes, and provider characteristics. For the free listing responses from consumers, a full list of these responses and the frequency with which they were reported will be compiled. The provider FGDs will be analyzed using methods described in the Design, Implementation, Monitoring and Evaluation (DIME) manual 1 [57]. Briefly, notes will be taken during discussions and then thematically coded using emergent coding. Notes and coding will be done in the local language and then summarized before translation.

\section{Planned secondary analyses}

We will examine changes in client symptom trajectories after delivery of each component of CETA (i.e., elevation, slope, and both elevation and slope), using the clinical monitoring form (symptom questions only in Table 2). To do this, we will use the modeling approach detailed by Singer and Willet [58] to examine changes in client symptom trajectories after delivery of each component of CETA (i.e., elevation, slope, and both elevation and slope). This approach involves adding timevarying dichotomous indicators representing whether each participant has completed each of CETA's components. This will allow us to look at whether clients' symptom levels significantly decreased (elevation) or whether they had significantly faster improvement (slope) immediately following completion of certain components. We will compare these changes in trajectories to monthly change in the control group participants.

We will further analyze the clinical monitoring from data (symptom questions only in Table 2 ) by using survival analysis to calculate the number of sessions that it takes for clients to achieve reliable change using the Reliable Change Index (RCI) [59]. Clients who end or drop out of treatment will be censored. We will use control group data to predict the number of sessions required to achieve improvement in the absence of intervention. We will then adjust for the difference between the average improvement slopes for each condition in the survival analysis.

\section{Data management and dissemination plan}

All data is collected via password-protected tablet using the CommCare mobile data collection service. Data is stored in a secure web-based portal. The data management team maintains a log of known data errors. Each week the data analyst exports the data to track eligibility and enrollment numbers and report to the larger study team. The investigators of the study will not perform any interim analysis. The primary outcome is the 6-month follow-up, so no impact analyses will be conducted prior to the completion of the trial.

Upon completion of the trial, analysis will be completed based on the aforementioned analysis plan using the full intent-to-treat sample. Results will be summarized for a final report to the funder and disseminated during meetings with Ukrainian government and NGOs, as well as, international NGOs who are working in the region. A plain language summary will be offered to community members and throughout public domains. Data will be stored on an encrypted drive and access will be granted to investigators who are added to the IRB as appropriate. De-identified data will be made available on request.

\section{Discussion}

This trial uses a robust design that allows us to compare the effectiveness of two interventions using a non-inferiority design. To our knowledge, this is the first trial evaluating a five-session modular, flexible, transdiagnostic treatment. We remained consistent with the cognitive-behavioral focus of this version of CETA by offering at least one cognitive and one behavioral element in each flow. The National Institute for Health and Care Excellence (NICE) guidelines have cognitive-behavioral therapy as one of the top recommendations for the effective treatment of depression, PTSD, and substance use disorders [60].

Rather than focus on elements that may be easier to teach, our approach was to "front-load" CETA with elements that appear to be strong mechanisms of action. Cognitive coping and restructuring is a key element in most evidence-based treatments for trauma, depression, anxiety and substance use $[9,24,50,51]$. Although often seen as a more "challenging" element, this cognitive skill is regularly taught to children and even preschoolers effectively [61] and has been used by lay providers with fidelity $[3,9,26,28,62]$. Exposure is a primary element in both PTS and anxiety disorders and is one of the 
most extensively used and studied elements in CBT [63]. This is often an element that providers may be anxious about implementing, and some providers initially believe it may be harmful [64] despite extensive evidence to the contrary [65-68]. We do recognize that these elements are likely to take longer for a provider to master compared to elements like Relaxation or Problem solving. Our rationale is that to obtain maximum symptom relief in a shorter time period, it may be necessary to have the strongest elements present.

This will also be the first trial directly comparing a brief versus standard course of CETA. While CETA demonstrated strong effectiveness on trauma, depression, and anxiety symptoms in the two completed trials $[26,28], 8-12$ sessions can be lengthy for some clients. An important empirical question is the level of effectiveness that can be obtained with shorter durations of treatment and practice of CBT skills, and whether this varies by type of presenting problems or client. This has important implications for further scale-up and sustainability of community-based psychotherapy services; namely, 'can we effectively reach more people faster, with potentially lower costs, to reduce population morbidity of common mental health problems?

The implementation of CETA in this study is different from the two completed trials in that we allow counselors more decision-making flexibility. For example, counselors are able to choose a primary problem of substance use, anxiety, depression or trauma whereas in the two completed trials all started with a trauma flow based on more restricted inclusion criteria. For those participants who are randomized to Standard CETA, providers have full flexibility in choosing the elements, order, and dosing that they provide after the first five sessions. We are also tracking the creation of flows by counselors, supervisors, and trainers separately to better understand the amount of training needed for providers to master clinical decision-making about client need and match with an element(s).

Our planned secondary analysis will explore how and when people recover by examining the impact of individual elements and length of time in treatment. Results from this study design will allow us to draw conclusions about the effects of both Brief and Standard CETA as well as about which elements are integral mechanisms of action, informing future implementation and fidelity efforts. In addition, our exploration of the implementation of CETA from the perspective of consumers and providers in the Ukrainian context, using a mixed-methods approach will inform the literature on Adoption, Acceptability, Appropriateness, Feasibility, and Accessibility/Reach of CETA. The quantitative measures being used will add to further refinement of implementation science measurement for low- and middle-income contexts.
A brief modular, flexible, transdiagnostic approach is particularly useful when working in settings with displaced and trauma-affected populations, given the high rates of comorbidity and high mobility in some settings. The results from this trial will inform future dissemination, implementation and scale-up of CETA in Ukraine as well as contribute to our understanding of these approaches in similar LMIC contexts.

\section{Trial status}

The trial is registered at ClinicalTrials.gov (NCT03058302; date of registration: 20 February 2017).

Recruitment began on 8 March 2017 and is expected to end on 1 June 2018.

\section{Additional file}

Additional file 1: Standard Protocol Items: Recommendations for Interventional Trials (SPIRIT) 2013 Checklist: recommended items to address in a clinical trial protocol and related documents*. (DOC $121 \mathrm{~kb}$ )

\section{Abbreviations}

ASSIST: Alcohol Smoking and Substance Involvement Screening Test; CBT: Cognitive-behavioral therapy; CETA: Common Elements Treatment Approach; D\&l: Dissemination and implementation; GPTSS: Global Posttraumatic Symptom Scale; HSCL-A: Hopkins Symptom Checklist-Anxiety subscale; IDSS: International Depression Screening Scale; JHU: Johns Hopkins University; LMIC: Low- and middle-income countries; NGO: Non-

governmental organization; NICE: National Institute for Health and Care Excellence; PTS: Post-traumatic stress; PTSD: Post-traumatic Stress Disorder; RCl: Reliable Change Index; SCID: Structured Clinical Interview for DSM; SCIDIV-RV: Structured Clinical Interview for DSM-IV-Research Version;

SPIRIT: Standard Protocol Items: Recommendations for Intervention Trials; USAID: United States Agency for International Development;

WHODAS: World Health Organization Disability Adjustment Schedule

\section{Acknowledgements}

The authors would like to thank our local research team as well as the current and future clients and families participating in the trial.

\section{Funding}

The study is supported by a grant from the United States Agency for International Development Victims of Torture Fund (USAID). Some authors are supported by the National Institutes of Health T32MH103210. The funder had no role in study design, data collection and analysis, interpretation of data, decision to publish, or preparation of the manuscript.

\section{Availability of data and materials}

The datasets generated and/or analyzed during the current study and other materials are available from the corresponding author on reasonable request.

\section{Authors' contributions}

LKM conceptualized the overall project and research design, leads the clinical protocol development, training, and supervision, is an active part of the study implementation, and led the drafting and completion of the manuscript. EH helped with the overall project and research design, is part of implementation, designed and oversees the measurement and statistical analyses, and helped with the manuscript. BD is part of implementation and contributed to the manuscript. NSS aided in the initial set up and

implementation and contributed to the manuscript. SB led the engagement of local organizations and recruitment, manages finances and staffing, is an active part of implementation, and contributed to the manuscript. JB helped with the research design, secured ethical approval at Johns Hopkins and contributed to the manuscript. SD helped with the research design, the clinical protocol development and training, and contributed to the 
manuscript. PB obtained funding, conceptualized the overall project and research design, directs the implementation and budgeting, and contributed to the manuscript. All authors read and approved the final manuscript.

\section{Ethics approval and consent to participate}

The study design and all study procedures were approved by the Institutional Review Boards at both Johns Hopkins University and the National University of Kyiv-Mohyla Academy. We will obtain informed consent from all participants in the study.

\section{Consent for publication}

Not applicable

\section{Competing interests}

The authors declare that they have no competing interests.

\section{Publisher's Note}

Springer Nature remains neutral with regard to jurisdictional claims in published maps and institutional affiliations.

\section{Author details}

'Department of Mental Health, Johns Hopkins Bloomberg School of Public Health, 624 N. Broadway, Baltimore, MD 21205, USA. ${ }^{2}$ Centre for Mental Health and Psychosocial Support, National University Kyiv-Mohyla Academy, Glasunova str 2/4, Kyiv 01042, Ukraine. ${ }^{3}$ Department of Psychology, University of Washington, Guthrie Hall (GTH), 119A 98195-1525, Seattle, WA 98105, USA. ${ }^{4}$ Department of International Health, Johns Hopkins Bloomberg School of Public Health, 624 N. Wolfe Street, Baltimore, MD 21205, USA.

Received: 19 December 2017 Accepted: 19 June 2018 Published online: 03 August 2018

\section{References}

1. Whiteford HA, Ferrari AJ, Degenhardt L, Feigin V, Vos T. The global burden of mental, neurological and substance use disorders: an analysis from the Global Burden of Disease Study 2010. PLoS One. 2015:10:e0116820.

2. Bolton P, Bass JK, Zangana GAS, Kamal T, Murray SM, Kaysen D, et al. A randomized controlled trial of mental health interventions for survivors of systematic violence in Kurdistan. Northern Iraq BMC Psychiatry. 2014;14:360.

3. Bass JK, Annan J, Mclvor Murray S, Kaysen D, Griffiths S, Cetinoglu T, et al. Controlled trial of psychotherapy for Congolese survivors of sexual violence. N Engl J Med. 2013;368:2182-91.

4. Rahman A, Malik A, Sikander S, Roberts C, Creed F. Cognitive behaviour therapy-based intervention by community health workers for mothers with depression and their infants in rural Pakistan: a cluster-randomised controlled trial. Lancet. 2008;372:902-9.

5. Neuner F, Onyut PL, Ertl V, Odenwald M, Schauer E, Elbert T. Treatment of posttraumatic stress disorder by trained lay counselors in an African refugee settlement: a randomized controlled trial. J Consult Clin Psychol. 2008;76: 686-94.

6. Ertl V, Pfeiffer A, Schauer E, Elbert T, Neuner F. Community-implemented trauma therapy for former child soldiers in Northern Uganda: a randomized controlled trial. JAMA. 2011;306:503-12.

7. Bolton $\mathrm{P}$, Bass J, Neugebauer $\mathrm{R}$, Verdeli $\mathrm{H}$, Clougherty KF, Wickramaratne $\mathrm{P}$ et al. Group interpersonal psychotherapy for depression in rural Uganda: a randomized controlled trial. JAMA. 2003;289:3117-24.

8. Patel V, Weiss HA, Chowdhary N, Naik S, Pednekar S, Chatterjee S, et al. Effectiveness of an intervention led by lay health counsellors for depressive and anxiety disorders in primary care in Goa, India (MANAS): a cluster randomised controlled trial. Lancet. 2010:376:2086-95.

9. Murray LK, Skavenski S, Kane JC, Mayeya J, Dorsey S, Cohen JA, et al. Trauma-focused cognitive behavioral therapy among trauma-affected children in Lusaka, Zambia: a randomized clinical trial. JAMA Pediatr. 2015; 169:761-9

10. Hensel-Dittmann D, Schauer M, Ruf M, Catani C, Odenwald M, Elbert T, et al. Treatment of traumatized victims of war and torture: a randomized controlled comparison of narrative exposure therapy and stress inoculation training. Psychother Psychosom. 2011;80:345-52.

11. Saxena S, Thornicroft G, Knapp M, Whiteford H. Resources for mental health: scarcity, inequity, and inefficiency. Lancet. 2007;370:878-89.
12. Patel V, Chowdhary N, Rahman A, Verdeli H. Improving access to psychological treatments: lessons from developing countries. Behav Res Ther. 2011;49:523-8

13. Kohn R, Saxena S, Levav I, Saraceno B. The treatment gap in mental health care. Bull World Health Organ. 2004;82:858-66.

14. McHugh RK, Barlow DH. Dissemination and implementation of evidencebased psychological interventions. Oxford: Oxford University Press; 2012.

15. Norton PJ, Barrera TL, Mathew AR, Chamberlain LD, Szafranski DD, Reddy R, et al. Effect of transdiagnostic CBT for anxiety disorders on comorbid diagnoses. J Depress Anxiety. 2013;30:168-73.

16. Norton PJ, Barrera TL. Transdiagnostic versus diagnosis-specific cbt for anxiety disorders: a preliminary randomized controlled noninferiority trial. J Depress Anxiety. 2012;29:874-82.

17. Dear BF, Staples LG, Terides MD, Karin E, Zou J, Johnston L, et al. Transdiagnostic versus disorder-specific and clinician-guided versus self-guided Internet-delivered treatment for generalized anxiety disorder and comorbid disorders: a randomized controlled trial. J Anxiety Disord. 2015;36:63-77.

18. Weisz JR, Chorpita BF, Palinkas LA, Schoenwald SK, Miranda J, Bearman SK, et al. Testing standard and modular designs for psychotherapy treating depression, anxiety, and conduct problems in youth: a randomized effectiveness trial. Arch Gen Psychiatry. 2012;69:274-82.

19. Sauer-Zavala S, Gutner CA, Farchione TJ, Boettcher HT, Bullis JR, Barlow DH. Current definitions of "Transdiagnostic" in treatment development: a search for consensus. Behav Ther. 2017:48:128-38.

20. Boustani MM, Gellatly R, Westman JG, Chorpita BF. Advances in cognitive behavioral treatment design: time for a glossary. Behav Ther (N Y N Y). 2017; 40:199-208.

21. Murray LK, Dorsey S, Haroz E, Lee C, Alsiary MM, Haydary A, et al. A common elements treatment approach for adult mental health problems in low- and middle-income countries. Cogn Behav Pract. 2014;21:111-23.

22. Weisz JR, Krumholz LS, Santucci L, Thomassin K, Ng MY. Shrinking the gap between research and practice: tailoring and testing youth psychotherapies in clinical care contexts. Annu Rev Clin Psychol. 2015;11:139-63.

23. Chorpita BF, Weisz JR, Daleiden EL, Schoenwald SK, Palinkas LA, Miranda J et al. Long-term outcomes for the Child STEPs randomized effectiveness trial: a comparison of modular and standard treatment designs with usual care. J Consult Clin Psychol. 2013;81:999-1009.

24. Chorpita BF, Daleiden EL, Weisz JR. Identifying and selecting the common elements of evidence based interventions: a distillation and matching model. Ment Health Serv Res. 2005;7:5-20.

25. Chorpita BF, Daleiden EL, Weisz JR. Modularity in the design and application of therapeutic interventions. Appl Prev Psychol. 2005;11(3):141-56.

26. Weiss WM, Murray LK, Zangana GAS, Mahmooth Z, Kaysen D, Dorsey S, et al. Community-based mental health treatments for survivors of torture and militant attacks in Southern Iraq: a randomized control trial. BMC Psychiatry. 2015;15:249

27. Glasgow RE, Fisher L, Strycker LA, Hessler D, Toobert DJ, King DK, et al. Minimal intervention needed for change: definition, use, and value for improving health and health research. Transl Behav Med. 2014;4:26-33.

28. Bolton P, Lee C, Haroz EE, Murray L, Dorsey S, Robinson C, et al. A transdiagnostic community-based mental health treatment for comorbid disorders: development and outcomes of a randomized controlled trial among Burmese refugees in Thailand. PLoS Med. 2014;11:e1001757.

29. Sijbrandij M, Bryant RA, Schafer A, Dawson KS, Anjuri D, Ndogoni L, et al. Problem Management Plus (PM+) in the treatment of common mental disorders in women affected by gender-based violence and urban adversity in Kenya: study protocol for a randomized controlled trial. Int J Ment Health Syst. 2016;10:44.

30. Chorpita BF, Daleiden EL. Mapping evidence-based treatments for children and adolescents: application of the distillation and matching model to 615 treatments from 322 randomized trials. J Consult Clin Psychol. 2009:77:566-79.

31. Internal Displacement Monitoring Center. IDMC: Ukraine Update. http://www. countries/countries/ukraine (2007). Accessed 5 July 2017.

32. Curran GM, Bauer M, Mittman B, Pyne JM, Stetler C. Effectivenessimplementation hybrid designs: combining elements of clinical effectiveness and implementation research to enhance public health impact. Med Care. 2012;50:217-26.

33. Chatfield A, Javetski G, Lesh N. CommCare evidence base 2015. https:// www.dimagi.com/wp-content/uploads/2015/02/CommCare-Evidence-BaseMarch-2015.pdf. Accessed 5 July 2018.

34. Haroz EE, Bass J, Lee C, Oo SS, Lin K, Kohrt B, Bolton P. Development and cross-cultural testing of the International Depression Symptom Scale (IDSS): 
a measurement instrument designed to represent global presentations of depression. Glob Ment Health. 2017;4:e17. https://doi.org/10.1017/gmh.2017. 16. eCollection 2017.

35. Haroz EE, Michalopolous L, Bolton PA, Bass JK. Development of new global instruments for assessing depression and post-traumatic stress related to torture and trauma. Washington, DC: Report to United States Agency for International Development; 2015.

36. WHO ASSIST Working Group. The Alcohol, Smoking and Substance Involvement Screening Test (ASSIST): development, reliability and feasibility. Addiction. 2002;97:1183-94. Blackwell Science Ltd

37. World Health Organization. The ASSIST Project-Alcohol, Smoking and Substance Involvement Screening Test. World Health Organization. http:// www.who.int/substance_abuse/activities/assist/en/. Accessed 5 July 2018.

38. Derogatis LR, Lipman RS, Rickels K, Uhlenhuth EH, Covi L. The Hopkins Symptom Checklist (HSCL): a self-report symptom inventory. Behav Sci. 1974;19:1-15.

39. Cardozo BL. Mental health, social functioning, and attitudes of Kosovar Albanians following the war in Kosovo. JAMA. 2000;284:569.

40. Kleijn WC, Hovens JE, Rodenburg JJ. Posttraumatic stress symptoms in refugees: assessments with the Harvard Trauma Questionnaire and the Hopkins Symptom Checklist-25 in different languages. Psychol Rep. 2001;88:527-32.

41. Lhewa D, Banu S, Rosenfeld B, Keller A. Validation of a Tibetan translation of the Hopkins Symptom Checklist 25 and the Harvard Trauma Questionnaire. Assessment. 2007;14:223-30.

42. Thapa SB, Hauff E. Psychological distress among displaced persons during an armed conflict in Nepal. Soc Psychiatry Psychiatr Epidemiol. 2005;40:672-9.

43. Ustun TB, Kostanjesek N, Chatterji S, Rehm J, World Health Organization. Measuring Health and Disability: Manual for WHO Disability Assessment Schedule (WHODAS 2.0). Geneva: World Health Organization; 2010. http://apps. who.int/iris/bitstream/10665/43974/1/9789241547598_eng.pdf?ua=1\&ua=1. Accessed 5 July 2018

44. Bolton P, Tang AM. An alternative approach to cross-cultural function assessment. Soc Psychiatry Psychiatr Epidemiol. 2002;37:537-43.

45. Doty B, Haroz EE, Singh NS, Bogdanov S, Bass JK, Murray LK, et al. Adaptation and testing of an assessment for mental health and alcohol use problems among conflict-affected adults in Ukraine. Confl Heal. 2018. https://doi.org/10.1186/s13031-018-0169-6.

46. First MB, Spitzer RL, Gibbon M, JBW W. Structured Clinical Interview for DSMIVTR Axis I Disorders, Research Version, Patient Edition. Revision: January 2010 [Internet]. Biometrics Research. New York: New York State Psychiatric Institute; 2002. Available from: http://www.scid4.org/info/refscid.html. Accessed 5 July 2018.

47. Liu X. Classification accuracy and cut point selection. Stat Med. 2012;31: 2676-86.

48. World Population Review: Ukraine. World Population Review, Walnut, CA, USA. 2017. http://worldpopulationreview.com. Accessed 5 July 2018.

49. Murray LK, Dorsey S, Bolton P, Jordans MJ, Rahman A, Bass J, et al. Building capacity in mental health interventions in low resource countries: an apprenticeship model for training local providers. Int J Ment Health Syst. 2011;5:30.

50. Foa EB. Prolonged exposure therapy: past, present, and future. J Depress Anxiety. 2011;28:1043-7.

51. Resick PA, Nishith $P$, Weaver $T L$, Astin MC, Feuer CA. A comparison of cognitive-processing therapy with prolonged exposure and a waiting condition for the treatment of chronic posttraumatic stress disorder in female rape victims. J Consult Clin Psychol. 2002;70:867-79.

52. Murray LK, Jordans MJD. Rethinking the service delivery system of psychological interventions in low and middle income countries. BMC Psychiatry. 2016;16:234.

53. Sloan JA. Assessing the minimally clinically significant difference: scientific considerations, challenges and solutions. COPD. 2009;2:57-62.

54. Guyatt GH, Osoba D, Wu AW, Wyrwich KW, Norman GR. Clinical Significance Consensus Meeting Group. Methods to explain the clinical significance of health status measures. Mayo Clin Proc. 2002;77:371-83.

55. Azur MJ, Stuart EA, Frangakis C, Leaf PJ. Multiple imputation by chained equations: what is it and how does it work? Int J Methods Psychiatr Res. 2011;20:40-9.

56. Rubin DB. Multiple imputation for nonresponse in surveys. Hoboken: Wiley; 2004.

57. Applied Mental Health Research Group. Design, implementation, monitoring and evaluation of cross-cultural trauma-related mental health and psychosocial assistance programs: A user's manual for researchers and program implementers. AMHR. 2013. Available from: https://www.jhsph. edu/research/centers-and-institutes/global-mental-health/resource- materials/design-implementation-monitoring-and-evaluation-dime/index. html. Accessed 1 Nov 2016.

58. Singer JD, Willett JB. Applied longitudinal data analysis. Oxford: Oxford University Press; 2003.

59. Jacobson NS, Truax P. Clinical significance: a statistical approach to defining meaningful change in psychotherapy research. J Consult Clin Psychol. 1991; 59:12-9.

60. NICE: The National Institute for Health and Care Excellence. London. 2017. https://www.nice.org.uk/guidance. Accessed 5 July 2018.

61. Cohen JA, Mannarino AP. A treatment study for sexually abused preschool children: outcome during a one-year follow-up. J Am Acad Child Adolesc Psychiatry. 1997;36:1228-35.

62. Kaysen D, Lindgren K, Zangana GAS, Murray L, Bass J, Bolton P. Adaptation of cognitive processing therapy for treatment of torture victims: Experience in Kurdistan, Iraq. Psychol Trauma. 2013:5:184-92.

63. Emmelkamp PMG. Anxiety and fear. Bellack AS, Herson M, Kazdin AE, Kazdin A. International handbook of behavior modification and therapy. 1990. 283-305.

64. Olatunji BO, Deacon BJ, Abramowitz JS. The cruelest cure? Ethical issues in the implementation of exposure-based treatments. Cogn Behav Pract. 2009; 16:172-80.

65. Tryon W. Possible mechanisms for why desensitization and exposure therapy work. Clin Psychol Rev. 2005:25:67-95.

66. Barrera TL, Mott JM, Hofstein RF, Teng EJ. A meta-analytic review of exposure in group cognitive behavioral therapy for posttraumatic stress disorder. Clin Psychol Rev. 2013;33:24-32.

67. Robjant K, Fazel M. The emerging evidence for Narrative Exposure Therapy: a review. Clin Psychol Rev. 2010;30:1030-9.

68. Frueh BC, Turner SM, Beidel DC. Exposure therapy for combat-related PTSD: a critical review. Clin Psychol Rev. 1995;15:799-817.

\section{Ready to submit your research? Choose BMC and benefit from:}

- fast, convenient online submission

- thorough peer review by experienced researchers in your field

- rapid publication on acceptance

- support for research data, including large and complex data types

- gold Open Access which fosters wider collaboration and increased citations

- maximum visibility for your research: over $100 \mathrm{M}$ website views per year

At BMC, research is always in progress.

Learn more biomedcentral.com/submissions 